\title{
Les plus anciens Vertébrés du Grand-Duché de Luxembourg : des Arthrodires Brachythoraci (Placodermi ; Dévonien inférieur)
}

\author{
The oldest vertebrates of the Grand-Duchy of Luxembourg: brachythoracid \\ artbrodires (Placodermi; Early Devonian)
}

\author{
Alain Blieck $^{\mathrm{a} *}$, Hervé Lelièvre ${ }^{\mathrm{b}}$, Dominique Delsate ${ }^{\mathrm{c}}$, Jacques Godefroid ${ }^{\mathrm{d}}$ \\ a Laboratoire de paléontologie et paléogéographie du Paléozoïque, URA 1365 CNRS, université des sciences et technologies de \\ Lille, Sciences de la terre, 59655 Villeneuve d'Ascq cedex, France \\ ${ }^{\text {b }}$ Laboratoire de paléontologie, URA 12 CNRS, Muséum national d'histoire naturelle, 8 rue Buffon, 75231 Paris cedex 05 , France \\ c Paléontologie, Musée national d'histoire naturelle de Luxembourg, rue Münster 25, 2160 Luxembourg-Grund, Luxembourg \\ 'Département de paléontologie, Institut royal des sciences naturelles de Belgique, rue Vautier 29, 1000 Bruxelles, Belgique
}

\begin{abstract}
Two dermal plates of the thoracic shicld of brachythoracid arthrodires have been discovered in Emsian localities of the Grand-Duchy of Luxembourg (GDL). They represent the first confirmed discovery of vertebrates in the Lower Devonian of the GDL, and thus become the oldest vertebrates of this country. They are also interesting by the fact that the occurrence of Brachythoraci in the Devonian of the Ardenne Allochthon has been, until now, very rare. The features of one of both plates fit rather well with the shape of the homologous plate of the Coccosteidae; this family is usually unreported in Lower Devonian rocks. (C Académie des sciences / Elsevier, Paris.)
\end{abstract}

Devonian / Emsian / Wiltz Formation / placoderms / Brachythoraci / Grand-Duchy of Luxembourg

Résumé - Deux plaques osseuses d'Arthrodires Brachythoraci (Vertébrés, Placodermes) conservées dans des schistes d'âge emsien sont décrites. Elles représentent la première mention confirmée de Vertébrés dans le Dévonien inférieur du Grand-Duché de Luxembourg et deviennent de fait les plus anciens Vertébrés de ce pays. Ces pièces sont également intéressantes en ce que les Brachythoraci sont particulièrement rares dans le Dévonien de I'Allochtone ardennais. Les caractéristiques de l'une des deux s'accordent assez bien avec celles de la plaque homologue des Coccosteidae, une famille de Brachythoraci habituellement non récoltée dans le Dévonien inférieur. (C Académie des sciences / Elsevier, Paris.)

Dévonien / Emsien / Schistes de Wiltz / placodermes / Brachythoraci / Grand-Duché de Luxembourg

\section{Abridged version}

The discovery of the specimens studied here is significant as it corresponds to the first confirmed record of Devonian arthrodire placoderms in the Grand-Duchy of Luxembourg (Delsate, 1997). Remains of arthrodires have been mentioned by Asselbergs (1913) and Lucius (1950) in the Schuttbourg 'quartzophyllades' (Lower Emsian; Maquil et al., 1984), and attributed to the genus Coccosteus. However, this assignment is probably wrong for two reasons: 1) Coccosteus has never been

\footnotetext{
Note présentée par Philippe Taquet.

Note remise le 2 février 1998, acceptée le 22 juin 1998.

*E-mail : alain.blieck@univ-lille1.fr 
found in the Lower Devonian; 2) at the beginning of this century, isolated bony plates of placoderms were often attributed to Coccosteus, which was referred to as a model.

The specimen ESQ 137 under study was collected in the 'Bois Woschend', north-east of Lellingen, near Wilwerwiltz (figure 1), in an invertebrate-bearing rock sample of the Upper Emsian, Wiltz Formation ('Grauwacke de Wiltz'; Maquil et al., 1984). This formation classically yields abundant invertebrate assemblages, among which brachiopods are noteworthy (Asselbergs, 1912; Bordet, 1939). The brachiopod genus Arduspirifer is represented by $A$. arduennensis arduennensis, which is typical for the Upper Emsian, Hierges Formation (sensu Godefroid et al., 1994) of the Ardenne Allochthon in Belgium (Polygnatbus laticostatus to P. serotinus conodont Zones).

The Lellingen placoderm was collected with Loreleiella dilatata (Roemer, 1844); chonetid indet.; 'Leptaena gr. rbomboidalis (Wilckens, 1769)'; Arduspirifer arduennensis arduennensis (Schnur, 1853); Paraspirifer sandbergeri Solle, 1971 ssp. indet.; Bracbyspirifer carinatus of. rbenanus Solle, 1971; Alatiformia sp. indet.; Subcuspidella sp. indet. A few bivalves were also recovered. Arduspirifer arduennensis arduennensis, Paraspirifer sandbergeri ssp. indet. and Brachyspirifer carinatus cf. rhenanus are consistent with an Upper Fmsian age for this sample (i.e. between -395 and -391 , or -388 and $385 \mathrm{My}$, according to recent isotopic data; Fordham, 1992; Odin, 1994; Gradstein and Ogg, 1996).

The morphological features of the specimen ESQ 137 (figure $2 B$ ) are those of a plate of the ventral trunk carapace of a brachythoracid arthrodire. It corresponds to a left anterior ventrolateral plate (AVL), with its ventral sensory groove (figures 3 and 4 ). Due to preservation conditions of this material, it fits approximately with the shape of a coccosteid
AVL (figure 3). However, because of the incompleteness of the specimen, it is determined as basal Eubrachythoraci gen. and sp. indet. (Eubrachythoraci sensu Lelièvre, 1995). As Coccosteidae are classically known in Middle Devonian strata (Denison, 1978), it would represent the first Emsian coccosteid or basal eubrachythoracid record. As another isolated plate from the Famennian of Esneux (Belgium), in the northeast Ardenne Allochthon, has also been compared to Coccosteidae (Lelièvre, 1982), this would mean that basal Eubrachythoraci may range from the Lower Devonian (Emsian) up to the Upper Devonian (Famennian) in the Ardenne Massif.

The second specimen $\mathrm{BQ} 117$ (figure $2 \mathrm{~A}$ ) was collected a long time ago and retrieved recently in the collections of the Musée national d'histoire naturelle de Luxembourg by one of us (D.D.). It comes from Hosingen, northeast of Lellingen, near the German border (Our River), most probably also from the Wiltz. Formation. It is a left anterior dorsolateral plate partially preserved in print, the ornamentation of which is composed of large, closely associated tubercles. It is larger than ESQ 137 , and the ornamentations of the two specimens are significantly different; thus, it is preferable to consider both plates as belonging to different taxa. One sensory line occurs on the dorsal face of $\mathrm{BQ} 117$ (sl, figure 2A). This pattern is shared, among Brachythoraci, by the Migmatocephala and the pachyosteomorphs (sensu Carr, 1991); on the contrary, basal eubrachythoracids have a different pattern with three sensory lines. Considering this sensory line disposition, the anterior dorsolateral plate BQ 117 meets the migmatocephalan and pachyosteomorph morphology. However, as this feature does not correspond to a shared derived feature, but to a primitive state, this plate is left in open nomenclature as Brachythoraci gen. et sp. indet.

\section{Introduction}

La découverte de deux plaques osseuses est importante, puisqu'elle représente la première mention confirmée de Placodermes dans le Dévonien du Grand-Duché (Delsate, 1997). Jusqu'à présent, une seule pièce de ce groupe y avait été signalée : Asselbergs (1913, p. 157), puis Lucius (1950, p. 58), ont mentionné l'existence de «Coccosteus " (en association avec des "plaques ganoïdes rhombiques ") dans les quartzophyllades de Schuttbourg d'âge Emsien inférieur [Quarzophylladen von Schüttburg (E 1b), unteres Ems, in Maquil et al., 1984]. Mais cette identification est probablement erronée, car aucun Arthrodire appartenant à ce genre n'est connu dans des niveaux du Dévonien inférieur. Au début du siècle, où peu de Placodermes avaient été décrits, le genre Coccosteus représentait le "modèle le plus fréquent " de Placoderme pour ces niveaux, et les auteurs rapportaient le plus souvent leurs découvertes de plaques isolées à Coccosteus, lui donnant ainsi une définition très large. Une révision du genre Coccosteus a été proposée par Miles et Westoll (1968), et des quelque quarante-quatre espèces créées avant cette révision, il n'en reste plus aujourd'hui que deux qui soient rapportées avec certitude au genre Coccosteus : $C$. cuspidatus de l'Eifélien d'Écosse et $C$. markae du Givétien d'Estonie (Dévonien moyen). Le cas de Coccosteus grossi (Obrucheva, 1962) du Dévonien moyen de la Plate-forme russe est plus délicat à régler, dans la mesure où les restes qui lui sont attribués ne sont pas diagnostiques du genre. Ils ne sont pas pris en considération ici. Les autres espèces sont, soit incluses dans des genres différents de Coccosteidae ou d'autres Arthrodires, soit placées en nomenclature ouverte (Miles et Westoll, 1968, p. 464-468).

\section{Matériel, localités et âge}

Les deux spécimens concernés sont conservés au Musée national d'histoire naturelle de Luxembourg sous les numéros de collection ESQ 137 et BQ 117 ; des moulages de ces deux pièces sont entrés dans les collections du Muséum national $d$ 'histoire naturelle de Paris, respectivement sous les numéros ARD 234 et 235 . Ces deux pièces sont conservées en empreintes (figure 2). La première se 


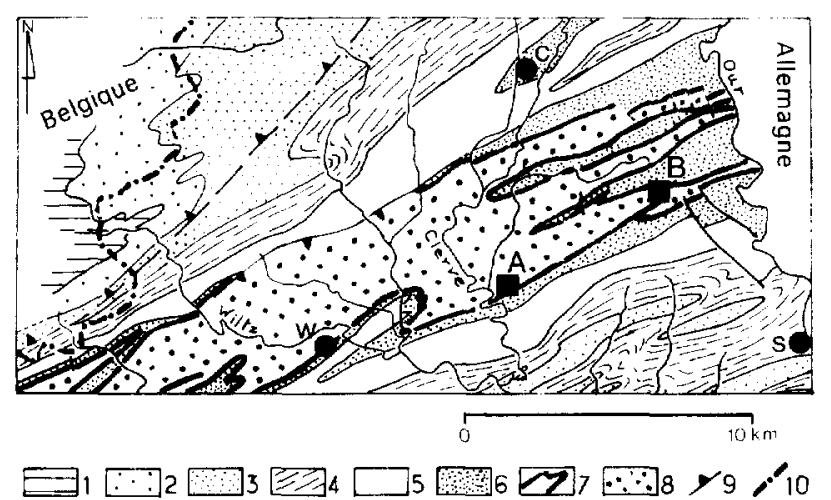

Figure 1. Emplacement des gisements de Lellingen (A) et $d^{\prime}$ Hosingen (B) dans le Nord du Grand-Duché de Luxembourg. Cadre géologique d'après Maquil et al. (1984, Abb. 1). Villes : c, Clervaux, s, Stolzenbourg (Stolzemburg), w, Wiltz. "Siegénien * : 1. Phyllades et quartzophyllades (Sg1). 2. Grès et schistes gréseux (Sg2). 3. Schistes gréseux grossiers (Sg3). Emsien : 4. Schistes de Stolzenbourg (Stolzemburg) (E1a). 5. Quartzophyllades de Schuttbourg (Schüttburg) (E1b). 6. Couches bigarrées de la Clerve (Clerf) (E2). 7. Quartzite de Berlé (q). 8. Schistes de Wiltz (E3). Autres figurés : 9. chevauchements, 10. Frontière politique.

Location of the Lellingen ( $A$ ) and Hosingen (B) fossiliferous sites in the northern Grand-Duchy of Luxembourg. Geological frame after Maquil et al. (1984, Abb. 1). Towns: C, Clervaux, s, Stolzenbourg (Stolzemburg), w, Wiltz. 'Siegenian': 1. Phyllites and 'quartzophyllades' $(\mathrm{Sg} 1)$. 2. Sandstones and sandy schists (Sg2). 3. Coarse-grained sandy schists (Sg3). Emsian: 4. Stolzemburg schists (E1a). 5. Schüttburg 'quartzophyllades' (E1b). 6. Clerf variegated beds (E2). 7. Berlé quartzite (q). 8. Wiltz schists (E3). other symbols: 9. Overthrusts, 10 . Political boundary. trouve dans un nodule de schiste argileux, dans lequel sont préservés également un fragment de Phacopidé (Trilobite) et une empreinte de Bivalve (ESQ 137, figure 2B). La seconde pièce est conservée dans le même type de sédiment et comporte des empreintes de Brachiopodes et de Bivalves (BQ 117, figure 2A).

Le spécimen ESQ 137 provient du Bois "Woschend » au nord-est du village de Lellingen, près de Wilwerwiltz. II a été récolté le long du talus d'un chemin forestier (figure 1) dans un schiste dur, gris-bleu foncé, truffè de restes d'Invertébrés. Il s'agit des Schistes (Grauwacke) de Wiltz, datés de l'Emsien supérieur [Schiefer von Wiltz (E3), oberes Ems, in Maquil et al., 1984].

Les Schistes de Wiltz, entre la frontière belge à l'ouest et la frontière allemande à l'est, ont livré plusieurs sites fossilifères dont les listes fauniques sont détaillées par Asselbergs (1912) et Bordet (1939, p. 29-30). De nombreux Invertébrés y ont été récoltés: Cnidaires (« COraux "), Crinoïdes, Brachiopodes, Trilobites, Bivalves, Gastéropodes, Nautilö̈des (Orthocères). Les Brachiopodes sont particulièrement abondants et représentés par les genres Platyorthis, Schizophoria, Strophodonta, Leptostrophia (Leptostrophiella), "Schellwienella", Chonetes (Chonetes), Chonetes (Plebejochonetes), Loreleiella, Oligoptycherhynchus, "Uncinulus", Arduspirifer, Brachyspirifer, Euryspirifer, Subcuspidella et Athyris [Asselbergs (1912) ; nomenclature actualisée]. Le genre Arduspirifer est représenté par $A$. Arduennensis arduennensis, taxon caractéristique de la formation (Grauwacke) de Hierges (sensu Godefroid et Stainier in Godefroid et al., 1994), Emsien supérieur du bord sud du synclinorium de
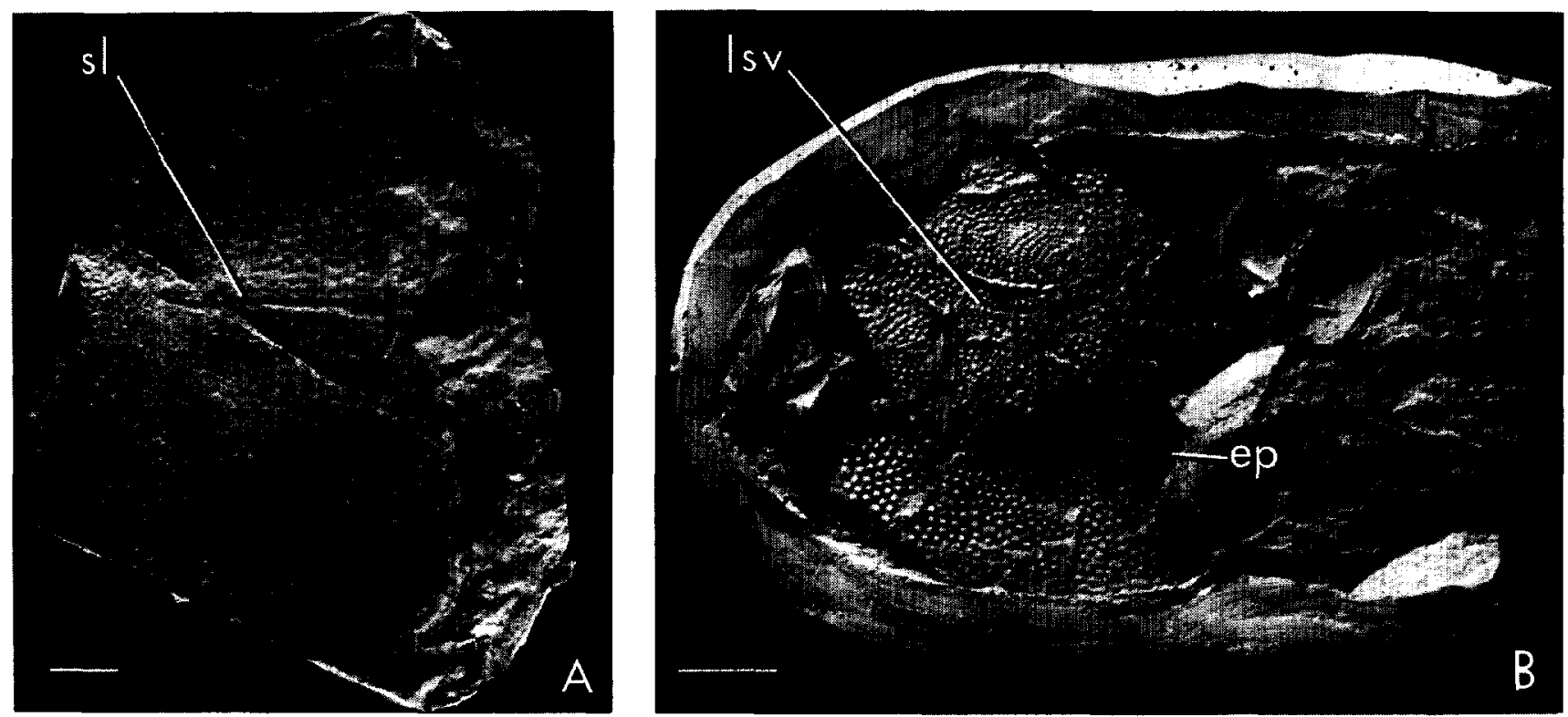

Figure 2. Vues externes des moulages en élastomère des spécimens BQ 117 et ESQ 137. A. Brachythoraci gen. et sp. indet., plaque dorsolatérale antérieure gauche (BQ 117). B. Eubrachythoraci basal gen. et sp. indet., plaque ventrolatérale antérieure gauche (ESQ 137). Abréviations : epémargination pectorale ; Isv- ligne sensorielle ventrale ; sl- sillon sensoriel principal. Echelles métriques : $1 \mathrm{~cm}$.

Elastomere casts in external views of the Grand-Duchy specimens BQ 117 and ESQ 137. A. Brachythoraci gen. et sp. indet., left anterior dorsolateral plate (BQ 117); B. Basal Eubrachythoraci gen. and sp. indet., left anterior ventrolateral plate (ESQ 137). Abbreviations: ep- pectoral incision; Isv- ventral sensory line; sl- main sensory line. Scale bars: $1 \mathrm{~cm}$. 
Dinant (Allochtone ardennais) en Belgique (biozones de Conodontes à Polygnathus laticostatus et à P. serotinus : Bultynck in Godefroid et al., 1994).

Les Brachiopodes du gisement de Lellingen, accompagnant ESQ 137, sont les suivants: Loreleiella dilatata (Roemer, 1844) ; Chonétidé indét. ; "Leptaena gr. rhomboidalis (Wilckens, 1769) "; Arduspirifer arduennensis arduennensis (Schnur, 1853); Paraspirifer sandbergeriSolle, 1971 ssp. indet. ; Brachyspirifer carinatus cf. thenanus Solle, 1971 ; Alatiformia sp. indet. ; Subcuspidella $\mathrm{sp}$. indet. Il y a également quelques Bivalves. Arduspirifer arduennensis arduennensis, Paraspirifer sandbergeri ssp. indet. et Brachyspirifer carinatus cf. rhenanus confirment un âge Emsien supérieur, soit entre -395 et -391, ou -388 et $-385 \mathrm{Ma}$ suivant les données radiochronologiques récentes (Fordham, 1992 ; Odin, 1994 ; Gradstein et Ogg, 1996).

Le spécimen BQ 117 provient de Hosingen, au nord-est de Lellingen, non loin de la frontière allemande (rivière Our). L'ancienne étiquette qui l'accompagne signale "Coblencien, Quartzite de Berlé ». Cependant, lithologiquement, il s'agit d'une roche à grain plus fin, très probablement des Schistes de Wiltz, sus-jacents au Quartzite de Berlé (A. Faber, comm. pers. à D.D.), à moins qu'il ne s'agisse des schistes intercalés à la base du quartzite (Maquil et al., 1984). Sur le terrain, quartzite de Berlé et schistes de Wiltz ne sont séparés que de quelques mètres (cf. Maquil et al., 1984) et le collecteur de l'époque pourra avoir confondu les deux. Les deux sites, Lellingen et Hosingen, peuvent donc être considérés comme presque synchrones (à l'échelle stratigraphique concernée). Ils sont situés à la bordure sud du Synclinorium de Neufchâteau-Eifel, dans l'Est du Grand-Duché (figure 1).

\section{Systématique}

Placodermi Mc Coy, 1848 - Arthrodira Woodward, 1891 - Brachythoraci Gross, 1932 - Eubrachythoraci Miles, 1971 emend. [Eubrachythoracei] - Eubrachythoraci basal gen. et $\mathrm{sp}$. indet. (figure $2 B$ )

Cette plaque (ESQ 137), fragmentaire et ornementée de tubercules étoilés, dont la taille augmente du centre de la plaque vers sa périphérie, appartient au plastron ventral de la cuirasse thoracique d'un Brachythoraci. C'est une plaque ventrolatérale antérieure gauche, dont aucun bord naturel n'est préservé. Cependant, la présence du sillon sensoriel ventral, dont le tracé en $V$ ouvert est caractéristique, permet de l'identifier. Les courbures, qui s'observent facilement sur un moulage, ont aussi servi à l'identification du spécimen et au repérage de la région de l'émargination pectorale (ep, figure 2B). Chez les Brachythoraci, cette plaque est en contact antérieur et antérolatéral respectivement avec les plaques intérolatérale et spinale, médialement avec les plaques médianes ventrales antérieure et postérieure. Postérieurement, un recouvrement caractéristique s'établit avec la plaque ventrolatérale postérieure (figure 3).
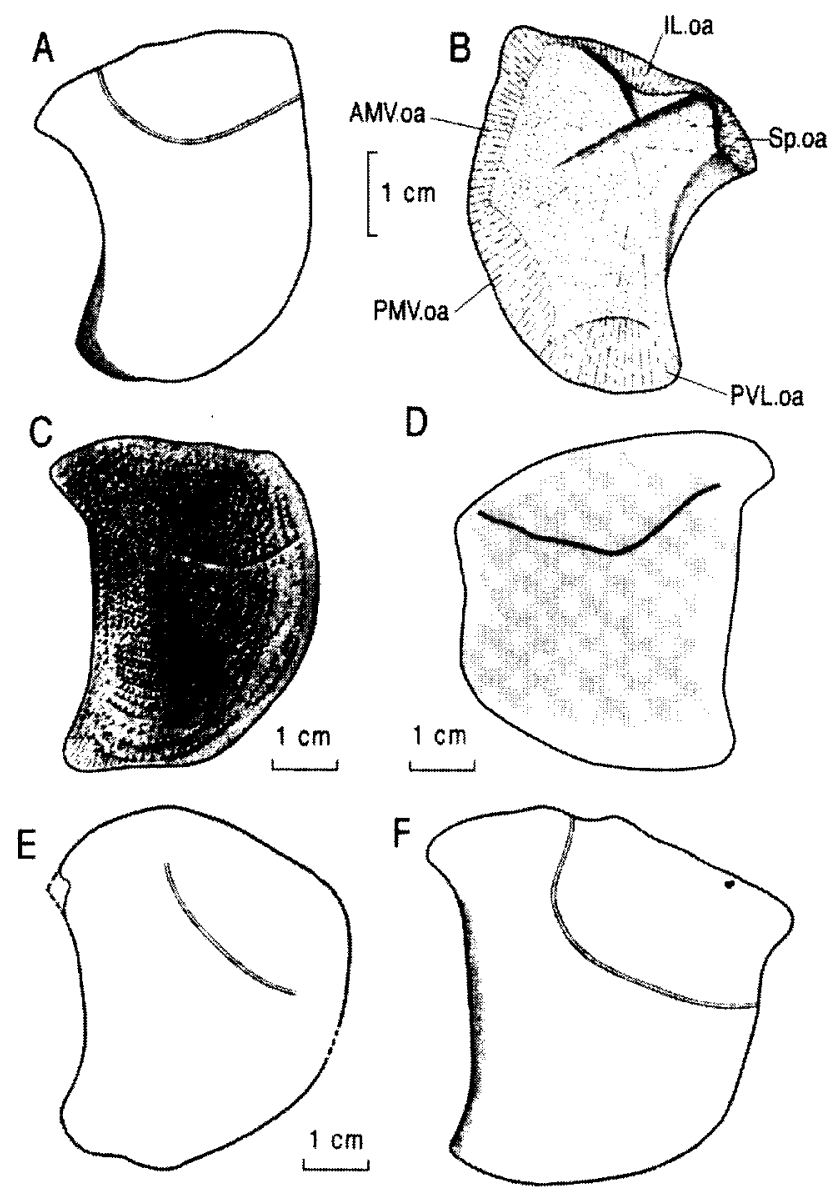

Figure 3. Diverses plaques ventrolatérales antérieures (AVL) d'Eubrachythoraci (A-C, E, F d'après Miles, 1966, figure 15). A, B, Coccosteus cuspidatus de I'Eifélien d'Ecosse. C. Dickosteus threiplandi de I'Eifélien d'Ecosse. D. Eubrachythoraci basal indet. de I'Emsien de Lellingen, G.-D. de Luxembourg. E. Plourdosteus mironovi du Frasnien de la Plate-forme russe. F. Plourdosteus canadensis du Frasnien du Canada (Québec, formation d'Escuminac) [A, C, E, F : AVL droites en vue externe (ventrale) ; B : AVL droite en vue interne (viscérale); $D$ : AVL gauche en vue externe (ventrale)]. AMV.oa, surface de recouvrement de la plaque médiane ventrale antérieure ; IL.oa, surface de recouvrement de la plaque intérolatérale ; PMV.oa, surface de recouvrement de la plaque médiane ventrale postérieure ; PVL.oa, surface de recouvrement de la plaque ventrolatérale postérieure ; Sp. oa, surface de recouvrement de la plaque spinale.

Various anterior ventrolateral (AVL) plates of Eubrachythoraci (A-C, E, F after Miles, 1966, figure 15). A, B. Coccosteus cuspidatus, Eifelian, Scotland. C. Dickosteus threiplandi, Eifelian, Scotland. D. Basal Eubrachythoraci indet., Emsian, Lellingen, G.-D. of Luxembourg; E. Plourdosteus mironovi, Frasnian, Russian platform. F. Plourdosteus canadensis, Frasnian, Canada (Quebec, Escuminac Formation) (A, C, E, F: right AVL in outer [ventral] view; B: right AVL in inner Ivisceral] view; D: left AVL in outer [ventral] view). AMV.oa, overlap area for the anterior median ventral plate; IL.oa, overlap area for the interolateral plate; PMV.oa, overlap area for the posterior median ventral plate; PVL.oa, overlap area for the posterior ventrolateral plate; $S p$. oa, overlap area for the spinal plate.

Le problème d'attribution de cette plaque à l'un des taxons de rang supraspécifique de Brachythoraci est posé par l'âge emsien des niveaux de Lellingen. En effet, si elle 
peut sans conteste être rapportée à un Arthrodire Eubrachythoraci basal, l'âge tendrait à l'attribuer à un Migmatocephala (sensu Lelièvre, 1995), même si l'attribution de cette plaque n'est pas dépendante de son âge. Les Migmatocephala sont le seul groupe monophylétique de Brachythoraci qui soit connu au Dévonien inférieur et dont certains membres sont caractérisés par la perte des plaques ventrolatérale postérieure et médiane ventrale postérieure. Cependant, la présence de la ligne sensorielle ventrale n'est pas constante chez les Brachythoraci basaux. Elle n'est actuellement pas signalée chez les Migmatocephala, alors qu'elle existe chez Maideria falipoui (Lelièvre, 1995) et chez les Buchanosteidae. Mais en tous les cas, lorsque cette ligne sensorielle est présente, elle est réduite à un court sillon profond. Cette disposition n'est pas celle du spécimen de Lellingen, sur lequel le tracé du sillon le rapprocherait plutôt du schéma qui existe chez les Coccosteidae (Eubrachythoraci). Par ailleurs, on observe aussi une tendance à la réduction de la longueur du plastron ventral de la cuirasse thoracique par le raccourcissement de la plaque ventrolatérale antérieure chez les Migmatocephala. On ne peut pas savoir si cette disposition est celle de ESQ 137. En effet, la surface interne postérieure de cette plaque n'est pas exposée et on ne peut constater si la surface de recouvrement avec la plaque postérieure est présente ou non.

À l'Eifélien (Dévonien moyen), d'autres formes de Brachythoraci, les Coccosteidae ou les Eubrachythoraci basaux, se sont développées; le plastron ventral de leur cuirasse thoracique ne présente pas de perte de plaque par rapport au plan d'organisation initial des Arthrodires. Or, d'après la figure 3, le contour général de notre spécimen $s^{\prime}$ accorde assez bien avec celui des plaques connues chez les Coccosteidae (figure 4).

Dans l'état actuel de préservation du spécimen de Lellingen et en l'absence d'autres éléments qui révéleraient la présence d'Arthrodires dans ce gisement, on ne peut pousser la détermination du spécimen plus loin. II s'agit donc d'un Eubrachythoraci basal gen. et $s p$. indet.

\section{Brachythoraci gen. et sp. indet. (figure 2A)}

Le spécimen BQ 117 (figure 2A) est une plaque dorsolatérale antérieure gauche tuberculée d'un Arthrodire Brachythoraci, conservée en empreinte. Elle n'est que partiellement préservée, puisque la région articulaire manque, ainsi que les surfaces de recouvrement dorsale et ventrale, respectivement pour les plaques médiane dorsale et latérale antérieure. II semble, cependant, que le bord postéroinférieur soit conservé. L'ornementation est composée de tubercules groupés de façon compacte; leur taille est supérieure à celle des tubercules de la plaque ESQ 137. Bien que différente de la précédente par ses plus grandes dimensions et par son ornementation, la plaque BQ 117 n'offre pas de critères d'attribution à un taxon autre que celui d'ESQ 137.

En effet, les plaques thoraciques dorsolatérales antérieures de Brachythoraci ne portent que peu de caractères diagnostiques. Chez les Eubrachythoraci basaux, les sillons sensoriels sont au nombre de trois, alors qu'il n'y a

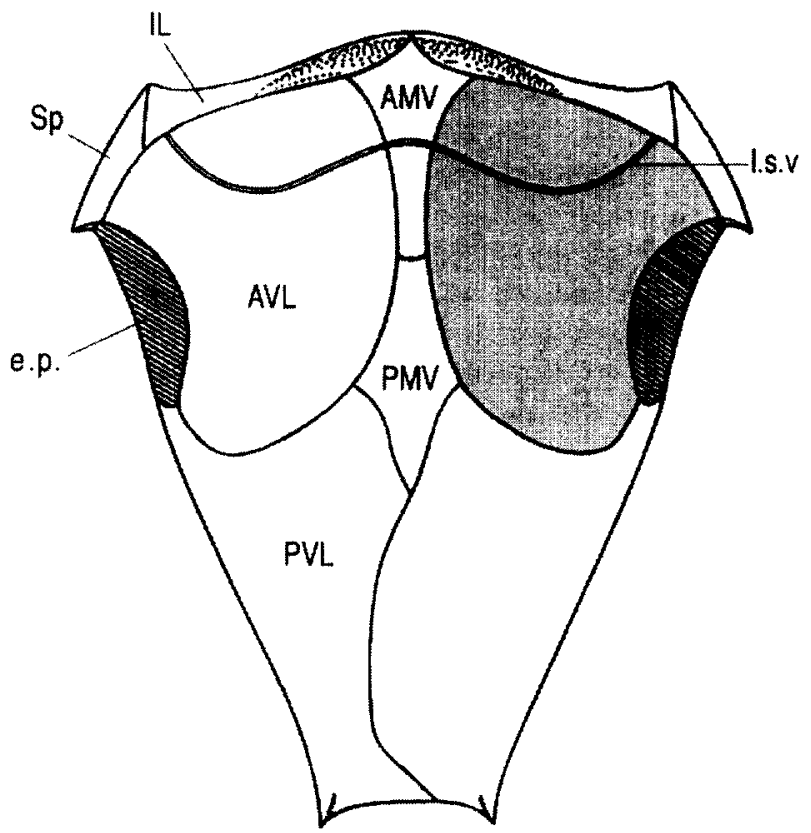

Figure 4. Vue ventrale de la cuirasse thoracique de Coccosteus cuspidatus d'après Miles (1966, figure 19A). La position du spécimen de Lellingen (ESQ 137) est replacée et indiquée en grisé. AMV, plaque médiane ventrale antérieure; AVL, plaque ventrolatérale antérieure ; e.p., émargination pectorale ; IL, plaque intérolatérale ; I.s.v, ligne sensorielle ventrale; PMV, plaque médiane ventrale postérieure ; PVL, plaque ventrolatérale postérieure; Sp, plaque spinale.

Ventral view of the trunk carapace of Coccosteus cuspidatus after Miles (1966, figure 19A). The Lellingen homologous plate (ESQ 137) is indicated in grey. AMV, anterior median ventral plate; $A V L$, anterior ventrolateral plate; e.p., pectoral emargination; IL, interolateral plate; I.s.v, ventral sensory line; $P M V$, posterior median ventral plate; $P V L$, posterior ventrolateral plate; Sp, spinal plate.

qu'un seul sillon, au trajet subhorizontal, chez le groupefrère des Eubrachythoraci : les Arthrodires Pachyostéomorphes (sensu Carr 1991, figure 19). Cependant, le schéma général à trois sillons sensoriels chez les formes basales d'Eubrachythoraci supporte quelques exceptions. Par contre, chez les Migmatocephala, la plaque dorsolatérale antérieure ne comporte qu'un sillon sensoriel dont le trajet occupe la même position que celui de BQ 117. Mais, prenant en compte l'absence de critères dérivés partagés, tant avec les Migmatocephala qu'avec les Eubrachythoraci basaux, nous laissons cette plaque du thorax d'un Placoderme Arthrodire en nomenclature ouverte.

\section{Revue taxonomique de quelques Placodermes du Dévonien de l'Allochtone ardennais (Nord de la France, Sud de la Belgique, Grand-Duché de Luxembourg)}

Plusieurs ordres de Placodermes sont diversement représentés dans le Dévonien de l'Allochtone ardennais. Ils 
sont connus depuis le siècle dernier. Une revue de détail des faunes de Vertébrés inférieurs du Paléozoïque a été proposée récemment par Blieck et Lelièvre (1995), Cloutier et Candilier (1995), Derycke et al. (1995). Nous ne donnons ici que les éléments principaux qui concernent les Placodermes. Au sein des Arthrodires Phlyctaeniina, quelques restes de Phlyctaeniidae, non déterminables au niveau générique, ont été signalés par Leriche (1948) dans le Dévonien inférieur (Praguien) de Wihéries en Belgique. Par contre, les Actinolepididae sont connus par divers restes (Blieck et Goujet, 1991 ; Goujet et Blieck, 1979), desquels émerge Bollandaspis woschmidti, un spécimen découvert dans une carotte du sondage de Bolland (Province de Liège). Ce spécimen est daté du Praguien supérieur ou de l'Emsien inférieur, et son état de conservation en fait un des Placodermes les plus complets découverts à ce jour (Schmidt, 1976). Comme on le constate, les Migmatocephala (Brachythoraci) sont inconnus pour l'instant dans le Dévonien inférieur de ces régions.

Au Dévonien moyen, alors que les Brachythoraci Coccostéides sont connus dans la plupart des célèbres gisements des Vieux Grès Rouges d'Europe, ils n'ont été signalés que par un spécimen dans une localité de Belgique : le Givétien du sondage de Tournai. II s'agit du crâne très bien conservé de Belgiosteus mortelmansi Lehman (1973). Au Dévonien supérieur (Famennien), la diversité des Placodermes à l'échelle mondiale se réduit; ce groupe de Vertébrés n'est plus représenté que par quatre taxa de rang supérieur, les Ptyctodontes, les Antiarches, les Phyllolépides et les Arthrodires (Brachythoraci et une famille de Phlyctaeniides, les Groenlandaspididae) (Carr, 1995). La présence des trois derniers taxa est signalée en Belgique, même si leurs restes, conservés dans des niveaux le plus souvent détritiques, sont peu abondants et souvent mal préservés. Pour les Brachythoraci, les découvertes ont été faites dans la Formation d'Esneux à Esneux (Lelièvre, 1982) et dans le Macigno d'Ouffet où ils voisinent avec les restes de l'Antiarche Bothriolepis lohesti (Leriche, 1931). Bothriolepis présente une répartition géographique mondiale (Long, 1984 ; G.C. Young, 1990). II est connu au Givétien-Frasnien (Dévonien moyen - supérieur), principalement sur les blocs continentaux laureuropéen et asiatique ainsi qu'en Australie-Antarctique (Gondwana Est), jusqu'au Famennien où sa répartition est encore plus vaste, puisqu'elle englobe une part plus importante du Gondwana (Amérique du Sud, Afrique du Sud et Australie) (Denison, 1978 ; Young, 1993).

\section{Commentaires}

Pour en revenir au Dévonien inférieur, les plaques osseuses de Lellingen et Hosingen constituent la première découverte confirmée de Vertébrés dans le Dévonien du Grand-Duché. La pièce mentionnée mais non figurée par Asselbergs (1913) et Lucius (1950) dans l'Emsien inférieur n'a pas été confirmée. Les plaques de l'Emsien supérieur de Lellingen et Hosingen sont donc, à l'heure actuelle, les plus anciennes découvertes de Vertébrés dans le GrandDuché.

La formation de Wiltz, qui a livré ces plaques, s'étend à I'ouest en Belgique et à l'est en Allemagne. En Belgique, la formation de Wiltz occupe la partie centrale du Synclinorium de Neufchâteau-Eifel, entre la frontière du GrandDuché et plus ou moins 8 à $10 \mathrm{~km}$ à l'est de Neufchâteau. Plus à l'ouest, elle disparaît, par suite du rétrécissement et de la terminaison du synclinorium (Asselberghs, 1946, p. 259, 444 et pl. 10). En Allemagne, les Schistes de Wiltz se retrouvent en bordure de synclinoria calcaires méridionaux de l'Eifel (Happel et Reuling, 1937, pp. 12-13; Struve, 1961, tableau 1 ; Mittmeyer, 1982, chart 2).

Leriche (1906, p. 39) cite, sans la figurer et sans indiquer la collection de dépôt, " une plaque ventro-latérale antérieure de Coccostéidé, probablement de Phlyctaenaspis » dans les Schistes de Wiltz. Cette mention pose plusieurs problèmes : $1^{\circ}$ ) Phlyctaenaspis est en fait synonyme de Phlyctaenius (Denison, 1978; V.T. Young, 1983); $2^{\circ}$ ) Phlyctaenius n'est plus classé avec les Coccosteidae (ibid.) ; et $3^{\circ}$ ) ceux-ci sont habituellement d'âge Dévonien moyen et supérieur, mais pas Dévonien inférieur (Denison, 1978 ; Carr, 1995). La détermination de Leriche est donc à prendre avec précaution et la remarque faite en introduction à propos du "Coccosteus» des Quartzophyllades de Schuttbourg est ici aussi valable : l'attribution d'une plaque osseuse isolée à un Coccosteidae doit être mise en doute. De plus, Leriche (1906) ne précise pas le site d'origine de la plaque; il parle des Schistes de Wiltz du Luxembourg, sans même dire s'il s'agit du Luxembourg belge ou du Grand-Duché. Cette mention n'a, à notre connaissance, jamais été confirmée. En Allemagne, des restes d'un Placoderme ont été récoltés dans la formation de Wiltz, près de Daleiden, à une dizaine de kilomètres à l'est de Clervaux (figure 1) et attribués à Phlyctaenaspis? sp. (Gross, 1937, p. 22-23, figure 12Q, PI. 2 : 10 ; Lippert, 1939, p. 38). Il s'agit en fait de la partie antérieure du moule interne de la face inférieure du toit crânien en vue dorsale d'un Arthrodire Actinolepina? (Actinolepina Miles sensu Denison, 1978, p. 46). D'autres restes de Vertébrés (Hétérostracés, Placodermes, Sarcoptérygiens) ont été récoltés dans cette région, à l'est de la frontière germano-luxembourgeoise (Gross, 1937 ; Lippert, 1939), mais dans des niveaux stratigraphiques différents, notamment dans les Couches de la Clerve (Klerfer Schichten) et dans l'équivalent des Quartzophyllades de Schuttbourg (Quartzophylladen von Schüttburg) (figure 1).

D'une façon générale, les restes de Placodermes sont rares au niveau mondial, comme le sont ceux des Vertébrés inférieurs dans leur ensemble (à l'exception de quelques gisements connus pour la diversité de l'assemblage fossile qu'on y récolte, notamment dans le Frasnien : Carr, 1995). Les découvertes de Lellingen et Hosingen sont donc un jalon significatif pour les Placodermes. Néanmoins, la relative rareté de ce taxon, même s'il constitue 
avec les Sarcoptérygiens le groupe dominant au Dévonien(" l'âge des poissons »), ne lui confère en général qu'une faible valeur comme outil biostratigraphique. Le synchronisme des assemblages est difficile à établir, même si quelques essais ont été avancés au niveau global (G.C. Young, 1974 ; Lelièvre et Goujet, 1986) ou au niveau régional (le Gondwana-Est par exemple : G.C. Young, 1988).

La cause majeure de l'incertitude de l'âge de nombreux assemblages de Vertébrés inférieurs aquatiques du Paléozoïque est l'absence ou la pauvreté du cortège co-occurrent d'Invertébrés à valeur stratigraphique. A Lellingen et à Hosingen, par contre, l'assemblage de Brachiopodes récolté permet de dater avec précision les Placodermes (Emsien supérieur). Cette association permet aussi de renforcer I'hypothèse d'un milieu marin pour ces Vertébrés. Sans entrer dans le détail, on rappellera que des Placodermes ont été à l'origine de l'hypothèse dulçaquicole pour le milieu de dépôt des Vieux Grès Rouges (VGR) du Dévonien d'Écosse et, par extension, du Dévonien mondial (Goujet in Blieck, 1985). Cette hypothèse a cependant été dénoncée comme trop exclusive et celle d'un milieu marin est désormais plausible pour plusieurs séries de type VGR ou, au moins, pour les niveaux à Vertébrés de ces séries (Goujet, 1984 ; Blieck, 1985 ; Blieck et lanvier, 1991). Le caractère marin de nombreux gisements classiques à Vertébrés du Silurien-Dévonien a d'ailleurs été confirmé par le biais de la géochimie (Schmitz et al., 1991) et de la micropaléontologie (Marshall et al., 1996).

\section{Conclusion}

La découverte, en apparence anodine, des spécimens de Lellingen et d'Hosingen porte en fait plusieurs particularités intéressantes. Elle est la première découverte de Vertébrés qui ait été confirmée dans le Dévonien du Grand-Duché et représente donc les plus anciens Vertébrés de ce pays. D'autre part, elle vient compléter notre information paléontologique sur les Placodermes de I'Allochtone ardennais, au sein de l'orogène paléozoïque d'Europe occidentale. Les spécimens sont datés de l'Emsien par le cortège de Brachiopodes ; cependant, la morphologie de l'un d'eux s'accorde à celle des Coccosteidae (Arthrodires Brachythoraci), classiquement connus à partir de l'Eifélien (Dévonien moyen; Denison, 1978). II représenterait donc la première découverte d'un Coccosteidé emsien, et cela dans l'Allochtone ardennais. Rappelons qu'une des plaques de Placodermes décrites dans le Famennien d'Esneux (Belgique), une plaque ventrolatérale postérieure (Lelièvre, 1982, figure 2C), présentait la particularité de posséder un processus postérieur latéral, considéré comme une synapomorphie des Coccosteidae. Mais, et c'est en cela que l'information est intéressante, cette famille n'était connue ailleurs que jusqu'au Frasnien (Eldenosteus, Janiosteus, Plourdosteus : Carr, 1995). L'Allochtone ardennais semble donc encore une fois présenter un particularisme quant à l'histoire des Placodermes : les Coccosteidae y seraient connus du Dévonien inférieur (Emsien) au sommet du Dévonien supérieur (Famennien).

Remerciements. Nous remercions J. Dercourt, D. Goujet et P. Taquet pour leurs remarques et critiques constructives sur le texte

\section{Références}

Asselbergs [sic] E. 1912. Contribution à l'étude du Dévonien inférieur du Grand-Duché de Luxembourg, Ann. Soc. Céol. Belg., 39 (1), Mém., M25-M112, pl. II-II

Asselbergs [sic] E. 1913. Le Dévonien inférieur du bassin de l'Eifel et de I'anticlinal de Givonne dans la région sud-est de l'Ardenne belge. En appendice : aperçu général sur le Bassin de l'Eifel entre la Meuse et la Kyll, Mém. Inst. Géol. Univ. Louvain, I, 1-175, pl. I-III

Asselberghs E. 1946. L'Eodévonien de l'Ardenne et des régions voisines, Mém. Inst. Géol. Univ. Louvain, 14, 1-598, pl. I-X

Blieck A. 1985. Paléoenvironnements des Hétérostracés, Vertébrés agnathes ordoviciens à dévoniens, in : Fischer ..-C. (éd.), Journées d'étude sur les indicateurs paléobiologiques de milieux, Bull. Mus. natt. Hist. nat., 4e sér., 7, section C, 2, 143-155

Blieck A. et Goujet D. 1991. Les Vertébrés du Dévonien inférieur d'Arville et de Nonceveux (Belgique), Ann. Soc. Géol. Nord, $2^{\mathrm{e}}$ série, $1(2), 67-78$

Blieck A. et Janvier P. 1991. Silurian vertebrates, in : Bassett M.G., Lane P.D. et Edwards D. (éds), The Murchison Symposium (Proc. Intern. Conf. Silurian System, Keele, 1989), Palaeontology, Special Paper, 44, 345-389

Blieck A, et Lelièvre H. 1995. Palaeozoic vertebrates of northern France and Belgium. Part I: Heterostraci, Osteostraci, Thelodonti, Placodermi (Devonian), in : Lelièvre H., Wenz S., Blieck A. et Clou- tier R. (éds), Premiers Vertébrés et Vertébrés inférieurs (VIII ème Congrès International, Paris, 4-9 septembre 1995), Geobios, Mém. Spéc. 19, 311-317

Bordet P. 1939. Note sur la faune de la Grauwacke de Wiltz (Emsien supérieur) dans le Grand Duché de Luxembourg, Mém. Soc. Sci. Nancy, 5-43

Carr R.K. 1991. Reanalysis of Heintzichthys gouldii (Newberry), an aspinothoracid arthrodire (Placodermi) from the Famennian of northern Ohio, with a review of brachythoracid systematics, Zool. J. Linn. Soc. London, 103, 349-390

Carr R.K. 1995. Placoderm diversity and evolution, in : Arsenault M., Lelièvre H. et Janvier P. (éds), Studies on Early Vertebrates (VIlth Intern. Symp., Miguasha Parc, Québec, 1991), Bull. Mus. nat. Hist. nat., Paris, $4^{\text {enne }}$ série, $17, \mathrm{C}(1-4), 85-125$

Cloutier R. et Candilier A.-M. 1995. Palaeozoic vertebrates of northern France and Belgium. Part III: Sarcopterygii (DevonianCarboniferous), in : Lelièvre H., Wenz S., Blieck A. et Cloutier R. (éds), Premiers Vertébrés et Vertébrês intérieurs (VIII ème Congrès International, Paris, 4-9 Sept. 1995), Geobios, Mém. Spéc., 19, 335-341

Delsate D. 1997. Un Arthrodire Brachythoraci (Placoderme) du Dévonien inférieur (Emsien) de Lellingen (Grand-Duché de Luxembourg). Note préliminaire, in : Maubeuge P.L. et Delsate D. (éds), Notes paléontologiques et biostratigraphiques sur le Grand-Duché de Luxembourg et les régions voisines, Trav. Scient. Mus. nation. Hist. nat. Luxembourg, 27, 1-15

Denison R. 1978. Placodermi, in :Schultze H.-P. (éd.), Handbook of Paleoichthyology, vol. 2, 128 p., 94 fig., G. Fischer Verlag, Stuttgart et New York 
Derycke C. Cloutier R. et Candilier A.-M. 1995. Palaeozoic vertebrates of northern France and Belgium. Part II: Chondrichthyes, Acanthodii, Actinopterygii (uppermost Silurian to Carboniferous), in : Lelièvre H., Wenz S., Blieck A. et Cloutier R. (éds), Premiers Vertébrés et Vertébrés inférieurs (VIII ème Congrès International, Paris, 4-9 septembre 1995), Geobios, Mém. Spéc. 19, 343-350

Fordham B.G. 1992. Chronometric calibration of Mid-Ordovician to Tournaisian conodont zones: a compilation from recent graphiccorrelation and isotope studies, Geol. Mag., 129 (6), 709-721

Godefroid J., Blieck A., Bultynck P., Dejonghe L., Gerrienne P., Hance L., Meilliez F., Stainier P. et Steemans P. 1994. Les formations du Dévonien inférieur du massif de la Vesdre, de la fenêtre de Theux et du synclinorium de Dinant (Belgique, France), Mém. Explic. Cartes Géol. Min. Belg., 38, 144 p., 80 fig., Bruxelles

Goujet D. 1984. Les Poissons Placodermes du Spitsberg : Arthrodires Dolichothoraci de la formation de Wood Bay (Dévonien inférieur), Cahiers de Paléontologie (Vertébrés), CNRS éd., Paris, 284 p., 114 fig., $30 \mathrm{pl}$.

Goujet D. et Blieck A. 1979. Les Vertébrés de l'Assise des Schistes et Grès de Pernes (Dévonien du Nord de la France), Ann. Soc. Géol. Nord, 98 (4), 263-278

Gradstein F.M. et Ogg J. 1996. A Phanerozoic time scale, Episodes, $19(1-2), 3-5,+$ insert chart

Gross W. 1937. Die Wirbeltiere des rheinischen Devons. Teil II, Abh. Preuß. Geol. Landesanst, Berlin, N.F., 176, 83 p., 29 fig., 10 pl.

Happel L. et Reuling H. T. 1937. Die Geologie der Prümer Mulde, Erläuterung zu einer geol. Karte $1: 25000$ (Senckenberg-Buch 5), Abh. senck. naturf. Ges., 438, 1-94, Pl. 1-10, Karte 1-4

Lehman J.-P. 1973. Un nouveau Coccostéomorphe, Belgiosteus mortelmansi, Ann. Paléont. (Vert.), 59 (1), 1-14

Lelièvre H. 1982. Ardennosteus ubaghsi n. g., n. sp., Brachythoraci primitif (Vertébré, Placoderme) du Famennien d'Esneux (Belgique), Ann. Soc. Géol. Belg., 105 (1), 1-7

Lelièvre H. 1995. Description of Maideria falipoui n. g., n. sp., a long snouted brachythoracid (Vertebrata, Placodermi, Arthrodira) from the Givetian of Maider (South Morocco), with a phylogenetic analysis of primitive brachythoracids, in : Arsenault M., Lelièvre H. et Janvier P. (éds), Studies on Early Vertebrates (VIIth Intern. Symp., Miguasha Parc, Québec, 1991), Bull. Mus. natl. Hist. nat., Paris, $4^{\text {e }}$ sér. 17, C (1-4), 163-207

Lelièvre $H$. et Goujet D. 1986. Biostratigraphic significance of some uppermost Devonian placoderms, in : Bless M.J.M. et Streel M. (éds), Late Devonian events around the Old Red Continent (Aachen, 1986), Ann. Soc. Géol. Belg., 109 (1), 55-59

Leriche M. 1906. Contribution à l'étude des Poissons fossiles du Nord de la France et des régions voísines, Mém. Soc. Géol. Nord, 5 430 p., 79 fig., $2+17$ pl., Lille

Leriche M. 1931. Les poissons famenniens de la Belgique. Les faciès du Famennien dans la région gallo-belge. Les relations entre les formations marines et les formations continentales du Dévonien supérieur sur la bordure méridionale du Continent Nord-Atlantique, Acad. Roy. Belg., Cl. Sci., Mém., $2^{e}$ sér., 10 (5), 72 p., 7 fig., 8 pl., Bruxelles

Leriche M. 1948. Sur la faune du Grès de Wihéries (Dévonien inférieur), Bull. Soc. Belge Géol., Paléont. Hydrol., 56 (3) [1947], $280-298$

Lippert H.J. 1939. Geologie der Daleider Mulden-Gruppe, Abh. senck. Naturf. Ges., Frankfurt a.M., 445, 66 p., 11 fig., 4 pl.
Long J.A. 1984. A plethora of placoderms: the first vertebrates with jaws? in : Archer M. et Clayton G. (éds), Vertebrate Zoogeography and Evolution in Australia, Hesperian Press, Perth, 185-210

Lucius M. 1950. Paläontologischer Charakter der verschiedenen Stufen des Unterdevons des CEslings, in : Geologie Luxemburgs. Das CEsling, Publ. Serv. Géol. Luxembourg / Veröffentlich. Luxemb. Geol. Dienst, Bd. VI, 54-62

Maquil R., Mosar J. et Thein 1. 1984. Unterdevon-Stratigraphie und variskischer Gebirgsbau im Eisleck / Nord-Luxemburg (Exkursion D am 26. und 27. April 1984), in : Geologische Exkursionen in der weiteren Umgebung von Echternach, Jber. Mitt. oberrhein. geol. Ver., N.F. 66, 57-75

Marshall J.E.A., Rogers D.A. et Whiteley M.J. 1996. Devonian marine incursions into the Orcadian Basin, Scotland, J. Geol. Soc. London, 153, 451-466

Miles R.S. 1966. Protitanichthys and some other coccosteomorph arthrodires from the Devonian of North America, Kungl. Svenska Vetenskapsakad. Handl., 10 (3), 5-49

Miles R.S. et Westoll T.S. 1968. The Placoderm fish Coccosteus cuspidatus Miller ex Ag., from the Middle Old Red Sandstone of Scotland. 1 : Descriptive Morphology, Trans. Roy. Soc. Edinburgh, 67, $374-476$

Mittmeyer H.-G. 1982. Rhenish Lower Devonian biostratigraphy, Cour. Forsch.-Inst. Senckenberg, 55, 257-269

Obrucheva O.P. 1962. Pantsirnye ryby devona SSSR (Kokkosteidy i dinikhtiidy) [Armoured fish from the Devonian of the USSR (Coccosteidi and Dinichthyidi)], Izdatelstvo Moskovskogo Universiteta, 1-189, fig. 1-42, pl. I-XIV [in Russian]

Odin G.S. 1994. Geological time scale (1994), C. R. Acad. Sci. Paris, 318, série II, 59-71

Schmidt Wo. 1976. Der Rest eines actinolepididen Placodermen (Pisces) aus der Bohrung Bolland (Emsium, Belgien), Mém. Explic. Cartes Géol. Min. Belg., 14, 23 p., Bruxelles

Schmitz B., Åberg G., Werdelin L., Forey P. et Bendix-Almgreen S.E. 1991. ${ }^{87} \mathrm{Sr} /{ }^{86} \mathrm{Sr}, \mathrm{Na}, \mathrm{F}, \mathrm{Sr}$, and $\mathrm{La}$ in skeletal fish debris as a measure of the paleosalinity of fossil-fish habitats, Geol. Soc. Amer. Bull., 103 (6), 786-794

Struve W. 1961. Zur Stratigraphie der südlichen Eifeler Kalkmulden (Devon: Emsium, Eifelium, Givetium), Senck. leth., 42 (3/4), $291-345$, pl. 1-3

Young G.C. 1974. Stratigraphic occurrences of some placoderm fishes in the Middle and Late Devonian, Newsl. Stratigr., 3 (4), 243-261

Young G.C. 1988. Antiarchs (placoderm fishes) from the Devonian Aztec Siltstone, southern Victoria Land, Antarctica, Palaeontographica, A, $202(1-4), 1-125$

Young G.C. 1990. Devonian vertebrate distribution patterns and cladistic analysis of palaeogeographic hypotheses, in: Mckerrow W.S. et Scotese C.R. (éds), Palaeozoic Palaeogeography and Biogeography (Symp. Oxford, 1988), Geol. Soc. London, Mem. 12, 243-255

Young G.C. 1993. Middle Palaeozoic macrovertebrate biostratigraphy of eastern Gondwana, in : Long I.A. (éd.), Palaeozoic Vertebrate Biostratigraphy and Biogeography, Belhaven Press, London, 9, 208-251

Young V.T. 1983. Taxonomy of the arthrodire Phlyctaenius from the Lower or Middle Devonian of Campbellton, New Brunswick, Canada, Bull. Brit. Mus. (Nat. Hist.), Geol, 37 (1), 1-35 